\title{
Evolução das variáveis teórico-empíricas interdisciplinares associadas ao design no contexto do desenvolvimento de interfaces para ambientes virtuais de aprendizagem
}

\author{
Evolution of the interdisciplinary theoretical and empirical \\ variables associated with the design in the development \\ of interfaces for virtual learning enviroments
}

Luana Maia Conti, Patrícia de Sá Freire, Simone Meister Bilessimo, Solange Maria da Silva

Interdisciplinaridade, O presente artigo apresenta os resultados da pesquisa exploratória realizada com design, design learning, ambientes virtuais de procedimentos de revisão sistemática da literatura em bases de dados eletrônicas, seguida de análise bibliométrica. Para isto, foram consideradas duas variáveis teóricoempíricas relacionadas ao desenvolvimento de interfaces para ambientes virtuais de aprendizagem e a influência do design neste processo, tendo como objetivo compreender a evolução e interdisciplinaridade destas variáveis, identificando escolas invisíveis, possíveis lacunas e oportunidades de futuras pesquisas. Entre outros elementos, a pesquisa apresenta em seus resultados uma relação direta entre design e as tecnologias no desenvolvimento de ambientes virtuais de aprendizagem, evidenciados em trabalhos interdisciplinares, das Áreas de Ciências da Computação, Engenharia e Ciências Sociais.

Interdisciplinarity, design, design learning, virtual learning environments.

This article presents the results of the exploratory survey of systematic literature review procedures in electronic databases, followed by bibliometric analysis. For this, were considered two variables (theoretical and empirical) related to the development of interfaces for virtual learning environments and the influence of design in this process, aiming to understand the evolution and interdisciplinarity of these variables, identifying invisible schools, possible gaps and opportunities future research. Among other conclusions, the research presents in its results a direct relationship between design and technology in the development of virtual learning environments, as evidenced in interdisciplinary work, from areas as Computer Science, Engineering and Social Sciences. 


\section{Introdução}

Em um contexto onde tudo se estabelece por meio da informação, é natural que se busque mecanismos que permitam gerenciá-la. Um exemplo é o posicionamento cada vez mais ativo do design. Ainda que pareça uma abordagem extremamente recente, há mais de duas décadas são feitas tentativas de diálogo entre design e educação apontando uma evidente importância de se observar o problema por meio da interdisciplinaridade. (Coutinho, 2006)

Neste sentido, é oportuno mencionar que o design é um importante artefato no processo de aprendizagem, podendo ser de grande utilidade já que, nos moldes contemporâneos, este processo não é mais dependente exclusivamente do professor, mas de fatores que possam despertar interesse no estudante.

Desde seu surgimento até hoje, ele manteve-se em natureza multidisciplinar e multifacetada - razão pela qual ainda é incompreendido em sua complexidade por muitas instâncias do conhecimento (Oliveira, Teixeira \& Maciel, 2006), dificultando sua caracterização. Ainda assim, Coutinho (2006) o defende como detentor das ferramentas adequadas à construção de mensagens visuais, que facilitam a compreensão do conteúdo - tanto a nível gráfico quanto informacional - e colabora para que ambiguidades sejam evitadas, otimizando todo o processo de compreensão de significado.

No caso do design instrucional, Romiszowski (2004) define-o como um esforço que envolve tanto princípios de comunicação, quanto de aprendizagem e ensino para a melhoria de materiais e ambientes, ao considerar as dimensões de uma aprendizagem realmente significativa do aluno - colocando esta última característica como sendo um desafio a ser vencido.

Por isso, é possível considerar que o papel do designer ganha abrangência no processo de evolução do mundo, já que o design ganhou nova significação (Coutinho, 2006). Em contraponto, torna-se corresponsável em uma nova proposta: gerar artefatos de modo culturalmente consciente e comprometido com os vieses e necessidades dessa nova sociedade.

Mesmo em consideração ao reconhecimento do design como importante constructo, o tema ainda é pouco explorado pela literatura científica. Nesse contexto, elabora-se a questão de pesquisa: como se processa a evolução dos estudos teóricos e empíricos interdisciplinares relacionados ao design no contexto do desenvolvimento de interfaces para ambientes virtuais de aprendizagem? Para responder a essa questão, definiu-se como objetivo desse estudo compreender a evolução, a interdisciplinaridade e as variáveis teórico-empíricas relacionadas ao design no desenvolvimento de ambientes virtuais de aprendizagem, identificando as escolas invisíveis, as possíveis lacunas e oportunidades de futuras pesquisas sobre o tema. 
Para o alcance deste objetivo, o artigo está assim estruturado. As duas próximas seções apresentam as definições de ambientes virtuais de aprendizagem por diferentes autores. Logo após, os procedimentos metodológicos são apresentados. De modo a auxiliar na compreensão do objeto de estudo, este artigo apresenta os resultados de uma revisão sistemática da literatura em bases de dados eletrônicas sobre as variáveis associadas, identificadas em estudos teóricos e empíricos.

Por fim, a análise da interdisciplinaridade do tema é mapeada e o artigo discorre acerca de implicações teóricas e práticas desses resultados e estabelece uma proposição de trabalhos futuros.

\section{Método}

Desde a segunda metade do século 20, devido ao amplo desenvolvimento tecnológico e infinidade de informações, vem-se sofrendo um complexo processo de transformação e reconstrução, até mesmo nas maneiras de ensinar e aprender. Aprofundar-se nestes novos processos é tão importante quanto difícil, mas observá-los sob o prisma das novas tecnologias, ambientes virtuais de aprendizagem e educação a distância pode ser um bom caminho.

Para compreender o que são Ambientes Virtuais de Aprendizagem é necessário um aprofundamento em suas raízes. Neste sentido, é inevitável a abordagem da Educação a Distância - cujo surgimento é impreciso, mas identificado, pelo menos, a partir do século 18, quando foi iniciada a dinâmica de cursos por correspondência. As novas possibilidades ofertadas pelos progressos científicos e o contexto social enfatizando, cada vez mais, a importância do conhecimento, ampliaram opções de cursos a distância com cenários midiáticos alinhados às possibilidades tecnológicas (relacionados principalmente à popularização da internet nos anos 80). Desta maneira, os cursos passaram a possibilitar acesso em qualquer lugar e hora nas modalidades de um para um, um para muitos, muitos para um e muitos para muitos. (Pereira, Schimitt \& Dias, 2007,p.5).

Bastos (2003) reforça esse entendimento, estabelecendo como principais características da EaD a separação geográfica, o vínculo a uma instituição educacional e a intermediação das Tecnologias da Informação e Comunicação - similar à concepção de Moran (2003) de 'processo de ensino-aprendizagem, mediado por tecnologias, onde professores e alunos estão separados espacial e/ou temporalmente' (Moran, 2003: 1). A figura 1 pode resumir, por exemplo, um histórico da Educação a Distância, destacando os principais meios utilizados para o processo de ensino-aprendizagem em cinco gerações bem definidas pelos autores: 
Figura 1 Cinco gerações da EaD - Fonte: adaptado de Moore e Kearsley (2007: 26)

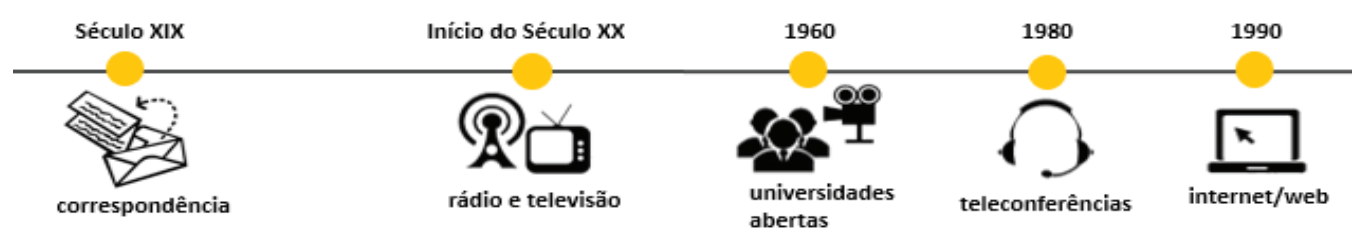

Para eles, as primeiras iniciativas de EaD se deram a partir do século XIX, por meio do envio por correio de materiais didáticos impressos. Em seguida, pelo rádio e, depois, com a televisão em transmissões de aulas. No final da década de 60, emergiram as universidades abertas com ampliação de acesso ao estudo que incluíam, entre outros meios, o uso do telefone e de fitas de vídeo. Quase vinte anos depois, no fim da década de 80, com um significativo avanço das tecnologias digitais, alunos e professores puderam se comunicar em tempo real por áudio e vídeo em conferências a distância. Neste momento, segundo os autores, experimentava-se a quinta geração de $\mathrm{EaD}$, caracterizada pelo uso do computador, internet e tecnologias multimídias; onde AVA's tornavam-se essenciais no sentido de oferecer espaços que disponibilizavam materiais didáticos e ofertavam ferramentas para conectar alunos e professores, síncrona e assincronamente, como chats e fóruns de discussão (Moore \& Kearsley, 2007).

Embora seja uma realidade relativamente recente, os ambientes virtuais de aprendizagem têm sido alvo de grande atenção, não faltando conceitos que buscam defini-los. Pereira, Schmitt e Dias (2007: 4), por exemplo, definem AVA's como ciber espaços aproveitados por mídias com o intuito de não somente difundir conteúdos, como propiciar interação e envolvimento entre atores do processo educativo.

Assim, pode-se dizer que ambientes virtuais de aprendizagem são espaços preparados com as mesmas intenções de um cenário físico de ensinar e aprender, porém estruturados essencialmente em meios virtuais específicos (mídias, hipermídias, textos, imagens etc.), visando aproximar alunos e professores, geográfica ou logisticamente distantes, para que estes alcancem o objetivo do processo de ensino-aprendizagem de construírem cooperativamente determinado conhecimento.

\section{3 design no contexto de desenvolvimento de AVA's}

Como pôde ser observado, o escopo das discussões acerca de ambientes virtuais de aprendizagem estende-se além dos limites da tecnologia em si e tem profundidade e abrangência maiores que a abordagem dos ambientes propriamente ditos. O mesmo acontece 
em relação ao design. O termo, que por si carrega um considerável conjunto de significados e interpretações - sendo frequentemente associado apenas à estética de um produto ou serviço - será aceito, neste trabalho, como disciplina que objetiva promover bem-estar na vida das pessoas e causar impactos positivos nas dinâmicas sociais, visualizando como problema quaisquer obstáculos que venham a prejudicar ou impedir a experiência (emocional, cognitiva, física ou estética) das pessoas, tomando como prioridade identificá-los para gerar soluções viáveis e eficazes (Vianna et al., 2013).

Diante da oferta crescente de informações, avanço efetivo e rápido das tecnologias, cobrança urgente pelo conhecimento, e importância de enfatizar a educação como ferramenta de transformação social, pode-se destacar ainda o argumento de Silva et al. (2014: 3), de que, cada vez mais, acredita-se que 'o caminho para o sucesso profissional, social e pessoal tem como base um tripé constituído de valores, ética e educação.' Ao unir estes universos tem-se o seguinte dilema: como gerenciar essas tecnologias e informações em um espaço virtual que propicie, da maneira mais eficaz possível, o processo de ensino-aprendizagem?

Neste sentido, duas abordagens principais acerca do design podem ser filtradas: design instrucional e design da informação, que segundo a Sociedade Brasileira de Design da Informação (SBDI, 2008: 29), entende-se por:

Área do design gráfico que objetiva equacionar os aspectos sintáticos, semânticos e pragmáticos que envolvem os sistemas de informação através da contextualização, planejamento, produção e interface gráfica da informação junto ao seu público alvo. Seu princípio básico é o de otimizar o processo de aquisição da informação efetivado nos sistemas de comunicação análogos e digitais.

Portanto, na prática, design da informação trata de conhecer o público-alvo de um determinado produto e as demandas de projeto envolvidas, a fim de compor sua natureza infográfica, dispondo as informações, de tal modo que sejam absorvidas com os níveis de prioridade definidos nos requisitos do projeto, respeitando padrões já instaurados de cognição, estética e semiótica, para facilitar sua apreensão por meio da oferta de uma experiência estéticocognitiva agradável.

No parâmetro atual, considerando o desenvolvimento de ambientes virtuais de aprendizagem, isso se dá, principalmente, por meio da interface de software; possibilidade proposta por volta de 1968 em protótipos de Doug Engelbart, apresentando um sistema passível de reunir informações posicionadas de maneira compreensível na tela de um computador (Johnson, 2001). Considerando sua utilidade, pode-se aludir a afirmação de Mari Junior (2012, p.12), que determina como principal papel da interface 'apresentar de maneira eficiente, ao usuário, o que ele pode e até mesmo o que ele deve fazer em cada ponto de um sistema'. O autor ainda dá uma importante contribuição ao enfatizar que o nível de envolvimento do usuário com a interface 
e as chances de processos interativos profundos se estabelecerem é proporcional ao conforto relativo às opções ofertadas na tela (Mari, 2012: 12).

Sob a ótica da educação, portanto, falar de interface é ainda mais importante, pois ela é o principal eixo de conexão entre o aluno e o conhecimento e é melhor aprofundada no design instrucional, definido por Filatro (2004, p.64) como:

Ação institucional e sistemática de ensino, que envolve o planejamento, o desenvolvimento e a utilização de métodos, técnicas, atividades, materiais, eventos e produtos educacionais em situações didáticas específicas, a fim de facilitar a aprendizagem humana a partir dos princípios de aprendizagem e instrução conhecidos.

Romiszowski (2004: 1) enfatiza essa concepção, descrevendo-o como área da tecnologia educacional relacionada ao "planejamento baseado em princípios científicos de comunicação, aprendizagem e ensino, visando a melhoria do processo ensino-aprendizagem e dos materiais didáticos elaborados.'. (Romiszowski, 2004); enquanto Ozcinar (2009) caracteriza design instrucional como multidisciplinar, identificando metas para o desenvolvimento de um sistema que atenda às necessidades de aprendizagem, incluindo materiais didáticos e atividades. Cybis et al. (2004, p.64) finalizam afirmando que os atributos de um sistema com fins educacionais devem sempre estar alinhados com as características do usuário, 'em termos cognitivos, culturais (hábitos), de competência (conhecimento e desempenho).' e às suas expectativas

Por conseguinte, embora possa ser interessante aos mais variados contextos, entende-se que, no processo complexo, peculiar e específico de ensino-aprendizagem há, não apenas aderência e aplicabilidade do design, mas também uma lacuna a ser desfiada por ele, que se estende tanto à gestão e planejamento de projeto como ao desenvolvimento infográfico de conteúdo e padrões estéticos com foco na experiência do usuário.

\section{Procedimentos metodológicos}

Esta pesquisa pode ser classificada como exploratória utilizando-se de procedimentos de revisão sistemática da literatura em bases de dados eletrônicas propondo-se a realizar uma análise bibliométrica.

\subsection{Revisão sistemática da literatura}

Para determinar a confiabilidade da pesquisa quanto aos seus resultados e análises, bem como, ser possível replicar seus procedimentos, foi utilizada a revisão sistemática da literatura e a analise bibliométrica. 
Em termos de pesquisa, alguns fatores são essenciais para o delineamento de um trabalho, tais como os métodos adotados em seu desenvolvimento. Assim, falar em método científico sistemático é adotar a compreensão de um objeto, a partir da construção de um 'quebra cabeças que permita o conhecimento do fenômeno como um todo. ' (Dagostin, Freire \& Guimarães Filho, 2014: 3). A Revisão Sistemática da Literatura (RSL) vem a ser, portanto, um método específico de pesquisa bibliográfica que objetiva revisar de forma planejada determinado assunto, a fim de responder a uma questão de pesquisa explícita (Dickson, 1999). Este processo, como assegura Freire (2013), aproveitado como procedimento exploratório, levanta dados por meio de rigorosas revisões de publicações acadêmicas em busca de evidências acerca de um tema, assunto ou tópico, enquanto possibilita a identificação, seleção e avaliação crítica destes estudos. Assim, é importante que a pergunta a ser respondida pela revisão esteja clara, bem como os critérios de inclusão e exclusão que determinarão os estudos a serem considerados - como cronologia, metodologia, autor e número de citações - e bancos de dados a serem utilizados.

\subsubsection{Planejamento}

Seguindo o procedimento, a pergunta da revisão sistemática é compreender a evolução das variáveis teórico-empíricas associadas ao design no contexto do desenvolvimento de interfaces para ambientes virtuais de aprendizagem. Para tal, primeiramente, estabeleceu-se duas principais variáveis envolvidas na pesquisa, hierarquizando-as de modo a estabelecer a variável "interface de ambientes virtuais de aprendizagem" como escopo geral, e a segunda, "design" uma menor abrangência, focando melhor o objeto.

Definida a Scopus como base de dados principal do estudo, iniciou-se a pesquisa buscando-se por interface de ambientes virtuais de aprendizagem, na qual foram alcançados 230 resultados. Acrescentando a segunda variável, no entanto, este número reduziu-se a 150. Desta maneira, alcançou-se 45 das 250 publicações iniciais processo que será melhor detalhado nas linhas seguintes.

\subsubsection{Estratégias da execução}

Conforme mencionado, foi informada no campo de busca a palavrachave da primeira variável, "virtual learning enviroments interface", resultando em 230 publicações. Acrescendo a segunda variável, o filtro de busca, "design", este número foi reduzido para 150 resultados.

No entanto, identificou-se que outros critérios poderiam ser aplicados a fim de reduzir os resultados e segmentar a pesquisa, de acordo com os objetivos do trabalho. Assim, foi aplicado como critério 
de exclusão a eliminação de quaisquer materiais que não estivessem relacionados às palavras chave: "e-learning", "virtual learning environments", "teaching", "user interfaces", "user computer interface" e "education", bem como publicações não relacionadas às seguintes áreas específicas: "computer science", "social sciences" e "engineering". Aplicados tais critérios de exclusão, reduziu-se cerca de $70 \%$ do resultado inicial, totalizando em 45 publicações. Estas se configuram na amostra desse estudo e, consequentemente, foram analisadas isoladamente a fim de compor uma síntese geral

\section{Apresentação e análise dos resultados}

Dentro da Revisão Sistemática da Literatura há três possibilidades de análise de resultados: análise descritiva (de natureza qualitativa), análise bibliométrica e meta análise - ambas quantitativas.

Macias-Chapula (1998: 134), tece uma definição robusta - embora de anos atrás - ao afirmar que bibliometria se trata do 'estudo dos aspectos quantitativos da produção, disseminação e uso da informação registrada' e, por meio dela, pode-se estabelecer padrões e modelos matemáticos, que possam medir tais processos, solidificando previsões e, até mesmo, apoiando a tomada de decisões em assuntos de pesquisa; enquanto Cunha (1985: 37) situa a bibliometria como um método que permite ao pesquisador encontrar um número restrito de publicações, que possam ser consideradas como mais relevantes em determinado tema.

\subsection{Uma visão histórica e geográfica dos estudos}

Por meio da análise bibliométrica identificou-se que a primeira publicação abarcando as variáveis mencionadas intitulava-se "NICE: Combining constructionism, narrative and collaboration in a virtual learning environment", de Roussos et al. (1997) - em Chicago, nos Estados Unidos da América - e objetivava apresentar um projeto de ambiente de aprendizagem participativo imersivo para jovens usuários, combinando a teoria construtivista e o desenvolvimento narrativo.

Contudo, é importante reiterar que, desde 1995 o assunto vem ganhando destaque: no período médio de dez anos, de 1996 a 2006, as publicações foram de cinco para dez. O mais relevante foi que, desde então, até 2011, em um período de pouco menos que seis anos, esta crescente triplicou, apresentando pico em 2011, com quase trinta relevantes trabalhos acerca do tema. Mesmo com a queda, as publicações do assunto não ficam abaixo de dez. Estas informações estão graficamente apresentadas na figura 2, a seguir. 
Figura 2 Fonte: Extraída da base de dados Scopus (2015)

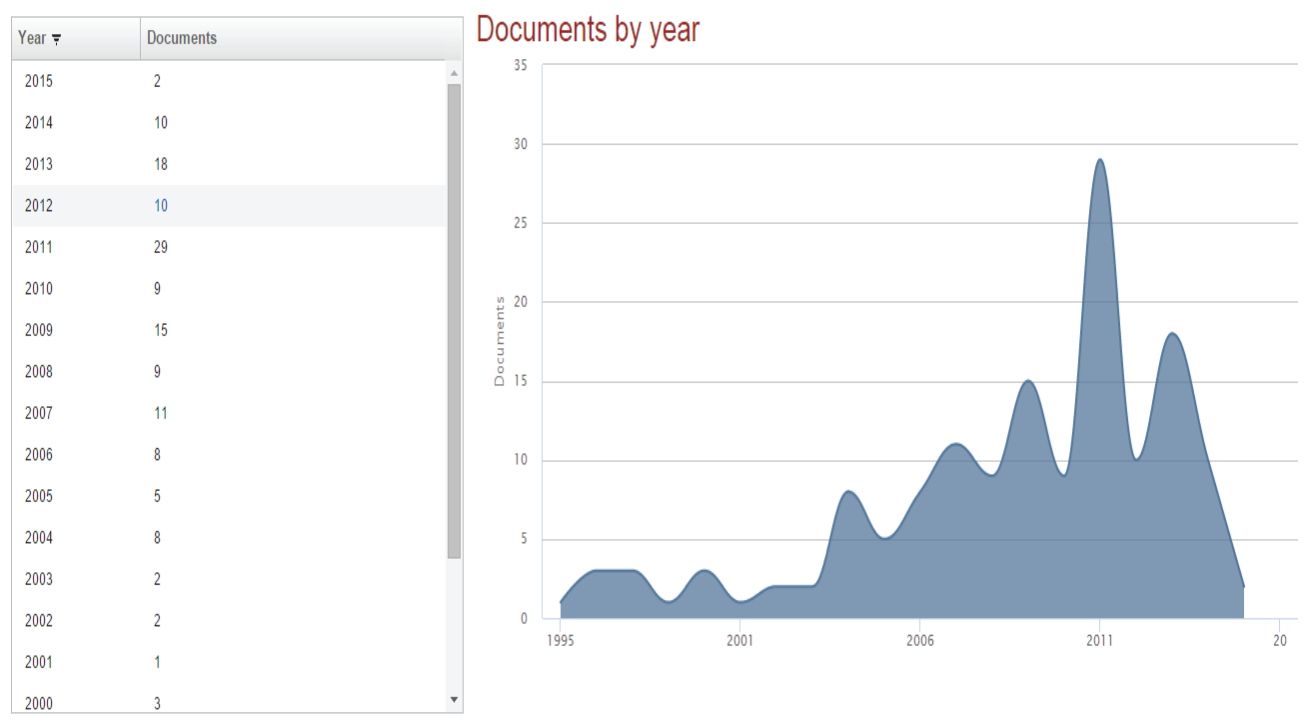

5.2 Descrição de periódicos, autores e áreas relacionadas

Dentre as publicações resultantes dos filtros e critérios de exclusão, pode-se mencionar especificamente algumas, cujo delineamento apresentou importantes contribuições para o caminho do diálogo entre as duas variáveis propostas neste trabalho, relacionadas no quadro 1.

Dentre elas, figura, por exemplo, o trabalho de Akeroyd (2005), que revisa a questão da interface em e-learning, bibliotecas digitais e conteúdo de aprendizagem com prospecções de cenários futuros.

Destaque também para Lyianage et al. (2013), que explora a eficácia de projetos WBL - sistemas educacionais para trabalhos baseados em aprendizagem - sob a perspectiva do aluno, apresentando cinco programas locais do ensino superior; e Klett (2004), que discute a questão de sistemas hipermídia, design para modelagem de aprendizagem, interface e correlatos, a fim de pensar em um sistema de atividades de aprendizagem que aumente a motivação individual e a performance.

Além destas, pode-se falar do estudo de Cole (2005), mostrando a usabilidade do AVA quanto à interface de software, usabilidade e aspecto funcional, além de comentários sobre motivação dos alunos, acessibilidade, design e funcionalidade das ferramentas de comunicação e metodologias de avaliação, que podem ser aplicadas a qualquer AVA.

Economou, Mitchell e Boyle (2000), por sua vez, publicaram estudo acerca da interatividade e dos problemas de comunicação social, que surgem na situação de aprendizagem, aproveitando como estudo de caso um projeto realizado por um museu de Manchester. Enquanto Georgouli (2011) apresenta uma visão geral dos ambientes 
virtuais de aprendizagem e esforços direcionados para o design de ambientes virtuais de aprendizagem adaptativos, mencionando os principais aspectos de um AVA e sistemas EaD, tecnologias de inteligência computacionais, integração de plataformas e gestão de aprendizagem.

Em uma abordagem mais lúdica, Harthoom e Hughes (2011) propõem brincadeira com fantoches como mecanismo para aumentar a presença dos alunos em um ambiente virtual de aprendizagem, além de algumas preocupações relacionadas ao método. Barzegar, Shojafar e Keyvanpor (2009) voltam-se especificamente para a importância da interface nos ambientes virtuais de aprendizagem, sugerindo que, mesmo com a variedade destas plataformas, ainda existem dificuldades quanto ao desenvolvimento de interfaces adequadas, motivadas pela falta de familiaridade com os princípios fundamentais em design de interface centrado no usuário. Por fim, nesta mesma abordagem, pode-se mencionar Humprhies-Smith (2009), na incorporação de um conteúdo em AVA, analisando a eficácia do processo na aprendizagem do conteúdo abordado.

Quadro 1 Publicações destacadas - Fonte: Elaborada pelas autoras

\begin{tabular}{llll}
\hline Título & Autor & Ano & Localidade \\
\hline $\begin{array}{l}\text { Requirements elicitation for virtual actors in } \\
\text { collaborative learning environments }\end{array}$ & $\begin{array}{l}\text { Economu, D. Mitchell, W.L.a, } \\
\text { Boyle, T. }\end{array}$ & 2000 & Reino Unido \\
\hline $\begin{array}{l}\text { The impact of user-centered design concepts in } \\
\text { virtual learning environments }\end{array}$ & Klett, F. & 2004 & Alemanha \\
\hline $\begin{array}{l}\text { Information management and e-learning: } \\
\text { Some perspectives }\end{array}$ & Akeroyd, J. & 2005 & Reino Unido \\
$\begin{array}{l}\text { Evaluation into the usability of a virtual } \\
\text { learning environment }\end{array}$ & Cole, l.; Lewis, D. & 2005 & Reino Unido \\
$\begin{array}{l}\text { User interface in virtual learning environment } \\
\text { Beyvanpour, M.R. }\end{array}$ & 2009 & Irã \\
\hline $\begin{array}{l}\text { Collaborative student centred learning } \\
\text { intellectual property for product designers }\end{array}$ & Humphries Smith, T. & 2009 & Reino Unido \\
$\begin{array}{l}\text { Design of educational systems for work based } \\
\text { learning (WBL): The learner experience } \\
\text { In virtual puppetry }\end{array}$ & $\begin{array}{l}\text { Penlington, R.; Casselden, B. } \\
\text { Virtual learning environments - An overview }\end{array}$ & Georgouli, K. & Reino Unido \\
\hline & Harhtoom, K., Hughes, S. & 2011 & Estados \\
\hline
\end{tabular}




\subsection{A elite de pesquisadores sobre o tema}

Os autores sensivelmente mais relevantes quanto à quantidade de publicações relacionadas ao conjunto das duas variáveis foram Derntl et al (2014), Dodero et al (2013) e Paracha et al (2009), como se pode visualizar no gráfico seguinte (Figura 3).

As publicações mais citadas, contudo, parecem oferecer informação de maior significância, visto que o trabalho de Van Raaij e Schepers, de 2008, "The acceptance and use of a virtual learning enviroments", foi mencionado 196 vezes, quase três vezes mais que o segundo mais citado, "Learning in a virtual world: experience with using Second Life for medical education", desenvolvido por Heyden et al (2010).

Figura 3 Publicações por autores Fonte: Extraída da base de dados Scopus (2015)

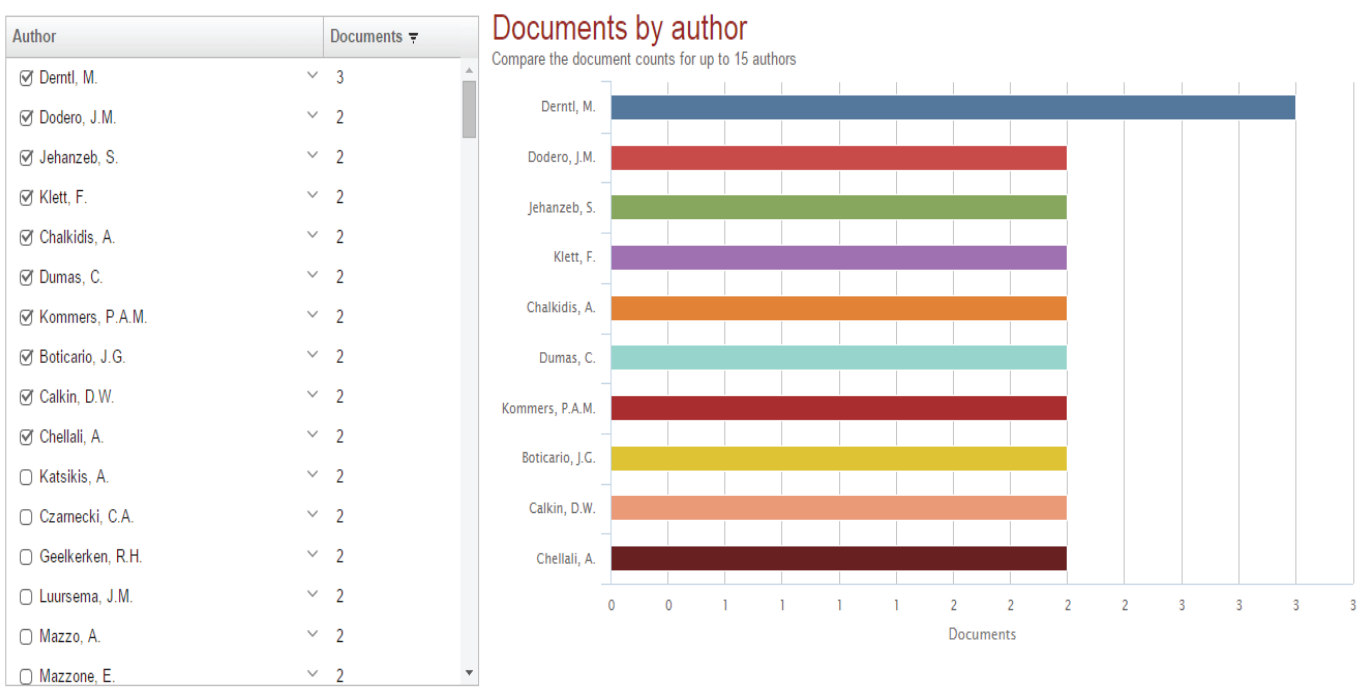

Outro dado interessante é o índice de publicação do tema por diferentes instituições de ensino e pesquisa, que mostra, por exemplo, o Brasil em quarta posição - atrás somente de países com forte representatividade tanto em pesquisa e inovação quanto em iniciativas de tecnologia e ensino, como Reino Unido, Estados Unidos e China, conforme gráfico, a seguir (Figura 4). 
Figura 4 Publicações por país Fonte: Extraída da base de dados Scopus (2015)

\begin{tabular}{|l|l|}
\hline Country/Territory & Documents ₹ \\
\hline$\square$ United Kingdom & 32 \\
$\square$ United States & 22 \\
$\square$ China & 12 \\
$\square$ Brazil & 11 \\
$\square$ Spain & 10 \\
$\square$ Germany & 8 \\
$\square$ Greece & 8 \\
$\square$ Australia & 4 \\
$\square$ Malaysia & 4 \\
$\square$ Belgium & 4 \\
$\square$ Taiwan & 4 \\
$\square$ Japan & 4 \\
$\square$ Austria & 3 \\
$\square$ Colombia & 3 \\
$\square$ France & 3 \\
$\square$ Netherlands & 3 \\
\hline
\end{tabular}

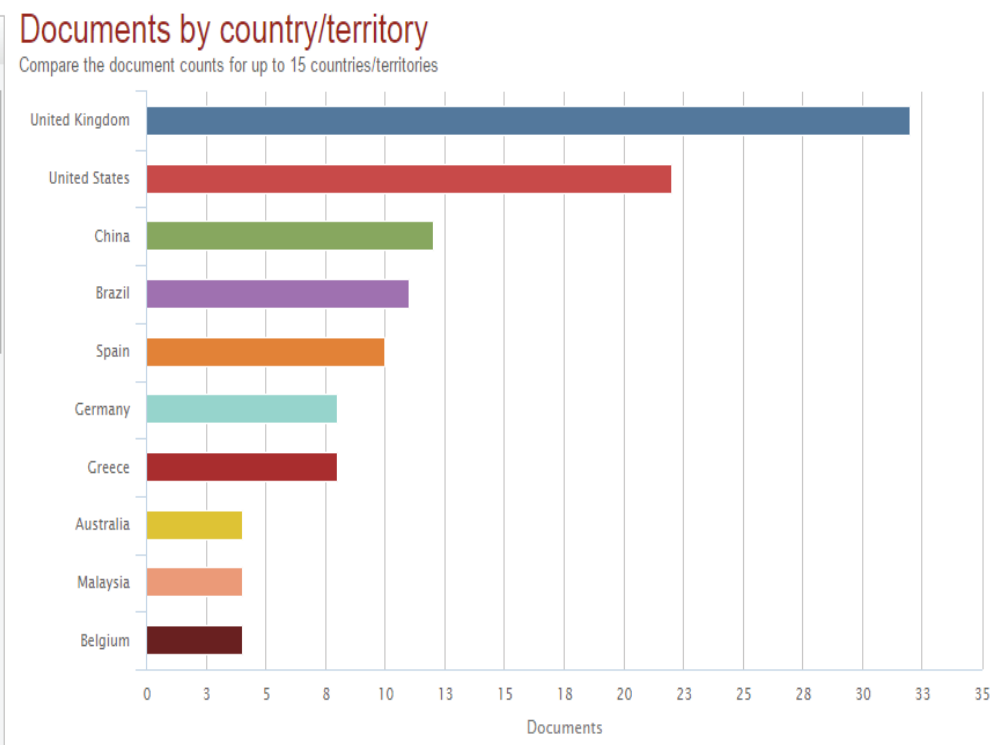

Ainda neste sentido, é possível dizer que foi identificada maior concentração de pesquisas relacionadas ao assunto, justamente nas áreas consideradas no delineamento deste trabalho (Figura 5): ciências da computação, engenharia e ciências sociais (nas quais se podem incluir aquelas relacionadas à comunicação, tais como pedagogia, psicopedagogia e psicologia, entre outras).

Figura 5 Publicações por área Fonte: Extraída da base de dados Scopus (2015)

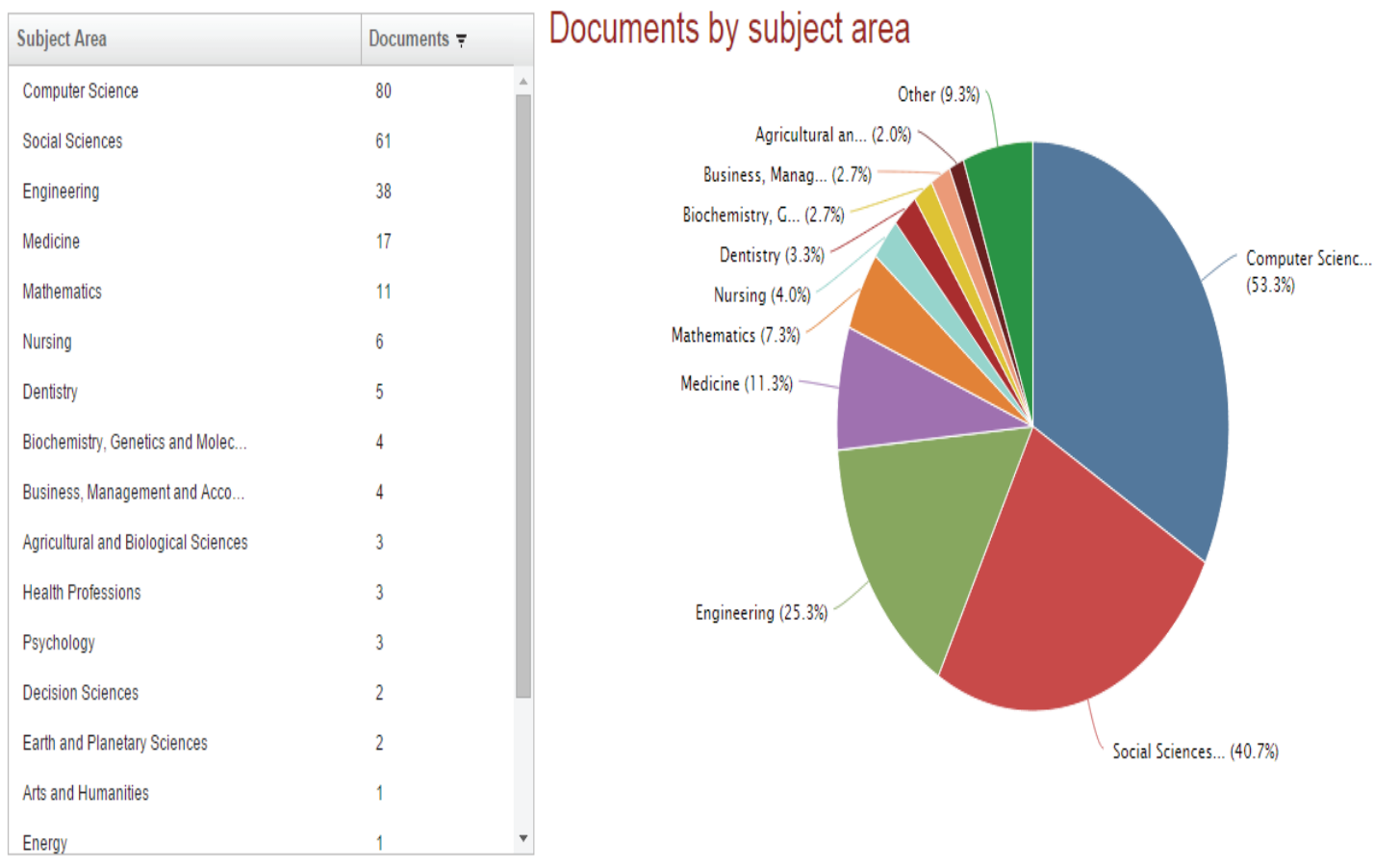




\section{Análise da interdisciplinaridade do tema}

Mediante resultados da pesquisa, identificou-se a interdisciplinaridade do tema, inclusive ao considerar isoladamente as variáveis. Além disso, verificou-se, por meio da análise bibliométrica, que autores das mais variadas áreas vêm buscando estudar a relação dos elementos abordados neste trabalho.

6.1 As variáveis teórico-empíricas propostas por área do conhecimento.

O gráfico seguinte (Figura 6) apresenta claramente as variáveis teórico-empíricas propostas por área de conhecimento e mostra a presença de estudos diversos relacionados a elas, considerando termos sobre ensino, realidade virtual, ambientes virtuais de aprendizagem, e-learning e ensino auxiliado por computador.

$\mathrm{Na}$ visualização gráfica evidenciaram-se as palavras-chave: "virtual learning enviroments" (ambientes virtuais de aprendizagem), como um dos termos mais incutidos na busca das variáveis teórico-empíricas, com 30\% de presença nos resultados; seguida pelo e-learning com $27 \%$ de resultados relacionados. Resultados relacionados à instrução auxiliada por computador (computer aided instruction), realidade virtual (virtual reality) e ensino (representado pela palavra teaching) vieram com menor destaque, mas ainda são considerados importantes para a análise das variáveis abordadas.

Figura 6 Publicação por termos Fonte: Extraída da base de dados Scopus (2015)

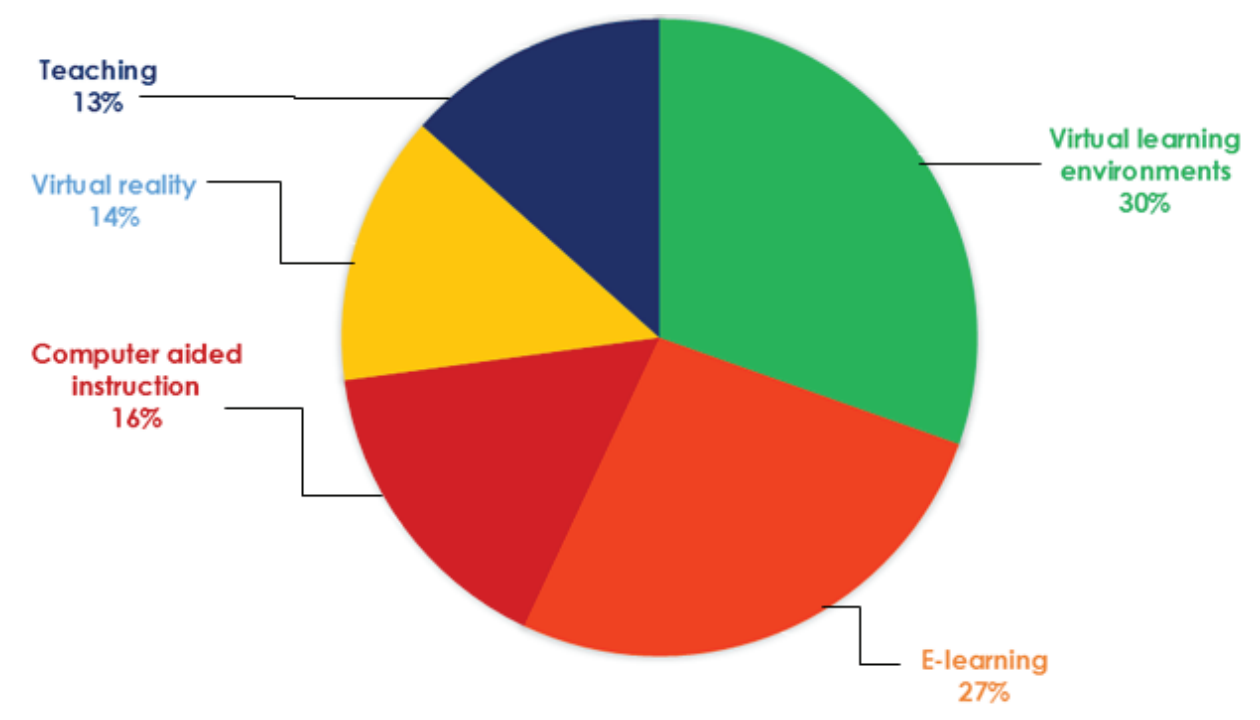




\section{Considerações finais}

Uma vez identificada a interdisciplinaridade e a evolução das variáveis teórico-empíricas acerca do design no desenvolvimento de interfaces de ambientes virtuais de aprendizagem, cabe elencar alguns possíveis estudos sobre o tema, como o aprofundamento de análises específicas de usabilidade por faixa etária, bem como o estudo de técnicas e ferramentas relacionadas às interfaces de ambientes virtuais de aprendizagem no âmbito do design centrado no usuário - tais como o levantamento dos métodos de investigação mais eficazes para cada etapa do desenvolvimento de plataformas deste tipo.

Outro aspecto importante é a crescente valorização do design na educação, refletido nos esforços para compreender seu impacto no processo de ensino aprendizagem e as diversas possibilidades que o diálogo entre disciplinas pode propiciar, alavancando novos métodos e ferramentas para este processo que precisa, cada vez mais, acompanhar as necessidades, preferências e requisitos do usuário. Neste sentido, cabe mencionar a importância das pesquisas brasileiras, tomando lugar de destaque em pesquisas de design voltado para educação.

A menção ao amplo leque de caminhos de pesquisa que podem surgir para esta finalidade é inevitável, assim como a importância de aprimorar objetos de estudo, a relação do design em ambientes virtuais de aprendizagem - incluindo psicologia e psicopedagogia - e a urgência em verificar até que ponto estes elementos impactam a qualidade e a eficiência dos processos de ensino aprendizagem - em ambientes virtuais e quaisquer outras práticas que utilizem interfaces humano-computador.

Além de, é claro, prospectar possibilidades de acordo com as tendências da tecnologia e da evolução dos processos relacionados, de modo a potencializar o ensino-aprendizagem, a partir da oferta de interatividade, posto que as tecnologias estão para servir o homem e a sociedade, e não há melhor modo de desenvolver ambos senão pelo ensino e cooperação - principalmente, em vias da sociedade do conhecimento.

\section{Referências}

AKEROYD, J. 2005. Information management and e-learning. Some perspectives. Aslib Proceedings: New Information Perspectives, v.57, n.2: 157-167.

BARZEGAR, S.; SHOJAFAR, M.; KEYVANPOUR, M.R. 2009. User Interface in virtual learning environment. International Conference for Internet Techonology and Secured Transactions, ICITST.

BASTOS, L. E. M. 2003. Avaliação do E-learning corporativo no Brasil. Dissertação (Mestrado) - Programa de Pós-Graduação Profissional em Administração, 
Escola de Administração da Universidade Federal da Bahia, Bahia. Disponível em: <http://www.adm.ufba.br/luis_eduardo2.pdf>. Acesso em: 22 set. 2004.

COLE, I.; LEWIS, D.; 2005. Evaluation of the usability of a virtual learning environment. In: 3rd International Conference of Education and Information Systems: Technologies and Applications, July 2005, Florida.

COUTINHO, S. 2006. Design da Informação para Educação. Infodesign: Revista Brasileira de Design da Informação, Rio de Janeiro, v. 1, n. 3: 49-60. Disponível em: <http://www.researchgate.net/publication/236672782>. Acesso em: 27 jul. 2015 .

CUNHA, M. V. 1985. Os periódicos em ciência da informação: uma análise bibliométrica. Ciência e Informação, Brasília, v. 14, n. 01: 37-45.

CYBIS, W., BERTIOL. A. H., FAUST, R. 2000. Ergonomia e Usabilidade Conhecimentos, Métodos e Aplicações. Florianópolis: Novatec.

DAGOSTIN, N.; FREIRE, P.S.; GUIMARÃES FILHO, L. P. 2014. Inovação para o desenvolvimento socioeconômico: Um estudo bibliométrico. In: Congresso Brasileiro de Excelência em Gestão, 10, 2014, Niterói. Anais. Niterói: [s.n.].

DERNTL, M; NEUMANN, S; OBERHUEMER; P. 2014. Lost in interaction in IMS learning design runtime environments. Educational Technology and Society, v.17, n.3: $332-342$

DICKSON R. 1999. Systematic Reviews In: Hamer S, Collinson G. Achieving evidence-based pratice: a handbook for practitioners. London: Baillière Tindall: $41-60$

DODERO, J.M; PALOMO-DUARTE, M.; RUIZ-RUBE, I. \& TRAVESSO, I. 2013. Semantic Accessibility to Elearning Web Services. Communications in Computer and Information Science: 258-265

ECONOMOU, D.; MITCHELL, L. W. \& BOYLE, T. 2000. Requirement elicitation for virtual actors in collaborative learning environments. Computers \& Education, v.34: 225-239.

FILATRO, A. 2004. Design Instrucional Contextualizado. São Paulo: Senac.

FREIRE, P. S. 2013. Aumente a Qualidade e Quantidade de Suas Publicações Científicas: Manual para elaboração de projetos e artigos científicos. Curitiba: Crv, $90 \mathrm{p}$.

GEORGOULI, K. 2011. Virtual Learning Environments - An Overview. In: Informatics (Pci), 2011 15th Panhellenic Conference On, Sept. 30 2011-Oct. 2 2011. 63-67.

HARTHOOM, K; HUGHES, S. 2011. Interface design to support situation awareness in virtual puppetry. Communications in Computer and Information Science: 112-115.

HEYDEN, R; STERNTHAL, E; MERIALDI, M. \& WIECHA, J. 2010. Learning in a virtual world: Experience with using Second Life for medical education. Journal of Medical Internet Research, v. 12, n.1.

JOHNSON, S. 2001. Cultura da Interface: Como o computador transforma nossa maneira de criar e comunicar. Rio de Janeiro: Jorge Zahar.

KLETT, F. 2004. The impact of user-centered design concepts in virtual learning environments. Proceedings of the Fifth Internacional Conference on Information Technology Base Higher Educacion and Training, ITHET: 222-226. 
LIYANAGE, L.; STRACHAN, R.; PENLINGTON, R. \& CASSELDEN, B. 2013. Design of educational systems for work based learning (WBL): the learner experience. Higher Education, Skills and Work-based Learning, v.3, n.1: 51-61

MACIAS-CHAPULA, C. A. 1998. O papel da informetria e dacienciometria e sua perspectiva nacional e internacional. Ciência da Informação, Brasília, v. 27, n. 2: 134-140, maio/ago.

MARI JÚNIOR, S. 2012. A linguagem visual nos processos interativos de interfaces gráficas em websites. Dissertação (Mestrado) - Curso de Programa de Mestrado em Comunicação Visual, Universidade Estadual de Londrina, Londrina.

MOORE, M \& KEARSLEY, G. 2007. Educação a distância: uma visão integrada. São Paulo: Thomson Learning, 2007.

MORAN, J. M. 2003. O que é educação a distância. Disponível em: < http://www2.eca.usp.br/moran/wp-content/uploads/2013/12/dist.pdf>. Acesso em: 18 ago. 2015

OLIVEIRA, A; TEIXEIRA, N. \& MACIEL, F. 2006. Design, educação, criatividade e as possibilidades de mediação dos processos de ensino e aprendizagem. In: Revista Design em Foco, v. 3, n.2, jul/dez 2006, Salvador: EDUNEB: 117-128.

OZCINAR, Z. 2009. The topic of instructional design in research journals: A citation analysis for the years 1980-2008. Australasian Journal of Educational Technology, v. 25, n. 4: 559-580. Disponível em: <http://www.ascilite.org.au/ ajet/ajet25/ozcinar.pdf >. Acesso em: 18 fev. 2014.

PARACHA, S.; JEHANZEB, S.; YOSHIE, O. 2009. Examining the theoretical schema of shimpai muyou! Narrative learning environment. Studies in Computational Intelligence, v. 231: 579-609

PEREIRA, A. T. C.; SCHMITt, V \& DIAS, M. R. A C. 2007. Ambientes Virtuais de Aprendizagem. In: PEREIRA, Alice T. Cybis. (orgs). AVA - Ambientes Virtuais de Aprendizagem em Diferentes Contextos. Rio de Janeiro: Editora Ciência Moderna Ltda.

ROMISZOWSKI, H. P. 2004. Avaliação no Design Instrucional e Qualidade da Educação a Distância: qual a relação?. Revista Brasileira de Aprendizagem Aberta e a Distância, São Paulo: 1-7, fev.

ROUSSOS, M., et al. 1997. NICE: combining constructionism, narrative and collaboration in a virtual learning environment. ACM SIGGRAPH Computer Graphics, v.31, n.3: 62-63.

SBDI, 2006. Sociedade Brasileira de Design da Informação. Disponível em: <http:// www.sbdi.org.br/>. Acesso: em 25 jul. 2013.

SILVA, A.; FERNANDES, M.C. ; SPANHOL, F. \& BASTOS, E. 2014. Design instrucional contextualizado em cursos on-line. In: congresso brasileiro de ensino superior a distância, 11., 2014, Florianópolis. Proceedings. Unirede: 1972 $-1983$.

VAN RAAIJ, E \& Schepers, J. 2008. The acceptance and use of a virtual learning environment in China. Computers \& Education: v.50, n.2: 838-852.

VIANNA, M.; VIANNA, Y.; ADLER, I.; LUCENA, B. \& RUSSO, B. 2013. Design Thinking: Inovação em negócios. 2. ed. Rio de Janeiro: Mjv Press. 


\section{Sobre os autores}

\section{Patrícia de Sá Freire}

<patricia.sa.freire@ufsc.br>

Campus Florianópolis

Departamento de Engenharia do Conhecimento do Centro Tecnológico/UFSC

Programa de Pós Graduação em Engenharia e Gestão do

Conhecimento

Universidade Federal de Santa Catarina

R. Eng. Agronômico Andrei Cristian Ferreira - Carvoeira, Florianópolis - SC, 88040-535.

\section{Solange Maria da Silva}

<solange.silva@ufsc.br>

Campus Araranguá

Curso de Tecnologias da Informação e Comunicação

Universidade Federal de Santa Catarina

Rodovia Governador Jorge Lacerda, 3201, Araranguá - SC, CEP 88905355 ,

\section{Simone Meister Sommer Bilessimo}

<simone.bilessimo@ufsc.br>

Campus Araranguá

Programa de Pós-Graduação em Tecnologias da Informação e Comunicação

Universidade Federal de Santa Catarina

Rua Pedro João Pereira, 150 Mato Alto - Araranguá - SC, CEP

88905-120.

\section{Luana Maia Conti}

<luanamconti@yahoo.com.br>

Campus Araranguá

Programa de Pós-Graduação em Tecnologias da Informação e Comunicação

Universidade Federal de Santa Catarina

Rua Pedro João Pereira, 150 Mato Alto - Araranguá - SC, CEP 88905-120.

Artigo recebido em 27/10/2015

Artigo aceito em 15/04/16 